dependence on temperature of radiation of heat from the same surface-namely, that the radiation is in proportion to the fourth power of the absolute temperature. This law was deduced originally from certain experiments of Prof. Tyndall on radiation from a heated platinum spiral (Pogo. Ann., Bd. cxxiv., quoted by Wüllner, "Exp. Physik," Bd. iii. I885). The law has been also considered by other writers, including Christiansen ( $A n n$. der Physik und Chemie, Bd. xix. 1883), and they have adduced experiments which seemed to them to confirm it.

The method of experimenting which I employ makes it easy to test the truth of such a law, and in fact to find the law, and I have accordingly made the necessary calculations for the former purpose.

In my experiments a current of known strength is passed through the wire under examination, and the increase in the resistance of the wire due to heating by the current is determined while the current is passing through it. When the temperature of the wire has become constant, the heat generated by the current (which can be calculated in absolute measure) must be equal to that emitted by the surface of the wire plus that lost at the ends of the wire by conduction. 'The temperature of the wire at the moment is also ascertained from its resistance (as was done by Siemens in his experiments on resistance of platinum wire at different temperatures, Proc.R.S., vol. xix. p. 443). I have recently been experimenting on platinum wires in a high vacuum down to about $\mathrm{I} / 20 \mathrm{M}$. (one twenty-millionth of an atmosphere), as was described to the British Association at its meeting at Aberdeen.

The results quoted in Table $I$. below were obtained with a straight platinum wire about half a metre long, $0.04 \mathrm{~cm}$. in diameter. It was contained in a glass tube about $0.6 \mathrm{~cm}$. in internal diameter, and was sealed into the tube at the two ends, the exhaustion being made by a small side tube. The exhaustion at the time of the experiment, as measured by a McLeod gauge, was I/ 5 M. The temperature of the room during the experiment was $15^{\circ} \mathrm{C}$.

The following two tables show the results of the experiment, and also a comparison of these results with the increase of emissivity with absolute temperature calculated according to Stefan's supposed law. Four cases have been taken which are numbered in the first column of each table. For these the current, C, and the resistance of the platinum wire, $R$, as found by experiment, are given in the second and third columns of Table I. The energy lost by the wire, $\mathrm{C}^{2} \mathrm{R}$, called $e$ in Table II., and the estimated temperature Centigrade are given in the fourth and fifth columns of Table I. The temperature of the surroundings at the time of the experiment was $15^{\circ} \mathrm{C}$. In the second table, the second, third, and fourth columns show the absolute temperatures of the wire and surroundings, and the energy lost, $e$, or $C^{22} \mathrm{R}$. Column 5 shows the ratios of the energy lost in the several cases to that lost in Case I, taken as unity. According to Stefan's law the heat emitted from the wire ought to be given by

$$
\mathrm{W}=\mathrm{A}\left(\mathrm{S}^{*}-\mathrm{T}^{*}\right),
$$

where $S$ is the absolute temperature of the wire, and $\mathrm{T}$ that of the surroundings. Hence if $S_{1}, T_{1}$ denote those temperatures in Case No. I, and if the heat emitted in this case be taken as unity, the heat emitted with any other temperatures, $\mathrm{S}$ and $\mathrm{T}$, would be

$$
\frac{\mathrm{S}^{4}-\mathrm{T}^{4}}{\mathrm{~S}_{1}^{4}-\mathrm{T}_{1}^{4}} \text {. }
$$

This ratio, for the temperatures of the several cases, is given in the sixth column of Table II.; and it will be seen by comparison with Column 5 of that table that the increase of loss of heat with increase of temperature does not follow any such exceedingly rapid law.
TABLE I.-Results of Experiment

$$
\begin{aligned}
& \text { Case Current Resistance } \begin{array}{c}
\text { Energy } \\
\text { emitted }
\end{array} \text { Tempera- Centi- Condition }
\end{aligned}
$$

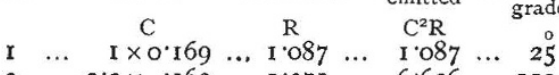

$$
\begin{aligned}
& \begin{array}{lllllllllll}
2 & \ldots & 2.2 & 2 & 169 & \ldots & 1 \cdot 371 & \ldots & 6.636 & \ldots & \text { 1 } 10
\end{array} \\
& \text { ( Wire per- }
\end{aligned}
$$

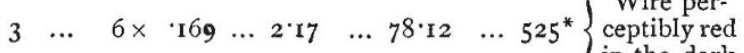

$$
\begin{aligned}
& \begin{array}{lllllllllll}
4 & \ldots & 6.5 \times & 169 & \ldots & 2.32 & \ldots & 98.06 & \ldots & 550
\end{array}
\end{aligned}
$$

* Temperature $525^{\circ}$ taken according to Draper's estimate of temperature

\begin{tabular}{|c|c|c|c|c|c|c|c|c|c|c|}
\hline Case & & $\begin{array}{l}\text { Absolute } \\
\text { tempera- } \\
\text { ture of } \\
\text { wire } \\
\text { (S) }\end{array}$ & & $\begin{array}{l}\text { bsolu } \\
\text { mper } \\
\text { e of } \\
\text { undin } \\
\text { (T') }\end{array}$ & & $\begin{array}{c}\text { Energy } \\
\text { emitted } \\
\text { (e) }\end{array}$ & & $\begin{array}{l}\text { Ratio } \\
e / e_{\mathrm{x}}\end{array}$ & & $\begin{array}{c}\text { Ratio } \\
S_{4}-T^{4} \\
S_{1}{ }^{4}-T_{x}\end{array}$ \\
\hline I & $\ldots$ & 298 & $\cdots$ & 288 & $\cdots$ & I.087 & $\cdots$ & I & $\cdots$ & I \\
\hline 2 & $\ldots$ & 383 & $\ldots$ & , & $\ldots$ & 6.636 & $\cdots$ & $6 \cdot 1$ & $\ldots$ & I6 \\
\hline 3 & $\ldots$ & 798 & $\ldots$ & , , & $\ldots$ & $78 \cdot 12$ & $\ldots$ & $7 I^{\circ} 9$ & .. & $438 \cdot 8$ \\
\hline 4 & $\ldots$ & 823 & $\ldots$ & , & $\ldots$ & 98.0 & $\ldots$ & $90 \cdot 2$ & $\ldots$ & $499 \cdot 8$ \\
\hline
\end{tabular}
of a body just visible in the dark.

TABLE II. - Comparison of Experimental Results with Calculation in accordance with Stefan's Laze

A comparison between the last two columns shows the enormous discrepancy between the results calculated from Stefan's law and those obtained by experiment.

I am now waiting for the use of a secondary battery, which I expect to have in a very short time, to determine the ratio between the energy lost at dull red heat, say $550^{\circ} \mathrm{C}$., and that lost at bright white heat ( $1200^{\circ} \mathrm{C}$. according to Draper), for the case of an incandescent lamp. Already, however, we know enough of the behaviour of incandescent lamps-for example, in the case of an eight-horse-power gas-engine, developing five-horsepower of electric energy, and feeding 50 sixteen-candlepower lamps-to be able to say that it does not require ten times as much work to keep the lamps at white heat as it does to keep them at dull red heat.

November 16

J. T. BOTTOMLEY

\section{ELLIPTIC SPACE}

FLLIPTIC geometry is more general than ordinary geometry. It refers to a three-dimensional space of a more general type than ordinary space. The ordinary mathematics supposes a more or less plausible assumption or axiom which reduces elliptic space to a special type. The present little paper is intended to illustrate the unartificial character of the elliptic geometry and to indicate the analytical nature of the axiom which the Euclidian geometry requires us to introduce. We investigate the measurement of distance on which the theory of elliptic space chiefly depends.

It is requisite to observe carefully the definitions which are made, and to refrain from the introduction of any notions not explicitly conveyed by the definition. Let us consider a "point" whose co-ordinates are $x_{1}, x_{2}, x_{3}, x_{4}$. It is not necessary to think of these co-ordinates as related to any geometrical scaffolding of tetrahedra or the like. It is in fact desirable to attach no geometrical import to the words, and merely to think of the word "point" as implying the four magnitudes just written. A second "point," $y$, will be similarly denoted by $y_{1} y_{2}$, $y_{3}, y_{4}$, and we define that the point $x$ is distinct from $y$, unless in the case where

$$
\frac{x_{1}}{y_{1}}=\frac{x_{2}}{y_{2}}=\frac{x_{3}}{y_{3}}=\frac{x_{4}}{y_{4}}
$$

If $\lambda$ be a numerical magnitude

$$
x_{1}+\lambda y_{1}, x_{2}+\lambda y_{2}, x_{3}+\lambda y_{3}, x_{4}+\lambda y_{4}
$$

will also denote a point, and then, it being assumed that $x$ and $y$ are distinct, we have a multitude of points corre- 
sponding to the various values of $\lambda$. This series we call a "straight line." But we here make no implication of any geometrical character attached to the line. It is merely a collocation of points, where each point is a group of four symbols and nothing more.

Each point on the line is thus correlated with a specific value of the numerical magnitude $\lambda$, and thus if we have two points we may refer to them as the points $\lambda$ and $\mu$ respectively.

As we are free from any geometrical meaning of our symbols, we can only introduce the expression "distance between two points" by defining precisely what is meant. The distance will be a function of $\lambda$ and $\mu$, whose form is to be decided by the properties which we desire to attribute to it. We may therefore select certain laws which we desire this function shall obey, and then discover what function will satisfy the conditions necessary.

We may take a hint from our familiar geometry as to the conditions to be imposed upon the distance function. If $A, B, C$ be three points upon a straight line, then there is no more fundamental notion of distance than that implied in the equation

$$
A B+B C=A C \text {. }
$$

We shall accordingly insist that our distance function shall be obedient to this law (which we may call Law I.). If, therefore, $\lambda, \mu, \nu$ be three points upon our straight line, and if $f(\lambda, \mu)$ denote the distance from $\lambda$ to $\mu$, then we find

$$
f(\lambda, \mu)+f(\mu, \nu)=f(\lambda, \nu),
$$

and as $\mu$ must disappear from this equation we have

$$
f(\lambda, \mu)=\phi(\lambda)-\phi(\mu) \text {. }
$$

The first step in the construction of an appropriate distance function has thus been taken, but we have still a wide range of indeterminateness, for $\phi(\lambda)$ may of course be any conceivable function of $\lambda$. It will therefore be competent for us to select some additional law and to insist upon obedience to it also.

Again we revert to our familiar geometry for a suggestion. In that geometry it is assuredly obvious that the distance between two points cannot be zero unless the two points are coincident. Trite as this condition may appear, it is yet sufficient to clear every trace of indeterminateness from the form of $\phi$ : we shall term this Law II.

Combining Laws I. and II. it will be easy to show that If $\boldsymbol{P}$ be a point on the line then there can only be one point, $Q$, on the line at a given distance from $P$, for, suppose that there was a second point, $Q^{\prime}$, then we have, by Law I.,

$$
P Q+Q Q^{\prime}=P Q^{\prime}
$$

but if $P Q$ be equal to $P Q^{\prime}$, then

$$
Q Q^{\prime}=\text { o, }
$$

from which, by Law II., we see that $Q$ and $Q^{\prime}$ must be identical.

If the point $P$ be defined by $\lambda$, and the point $Q$ at a given distance therefrom be defined by $\mu$, then the relation between $\lambda$ and $\mu$ must be of the one-to-one type. The distance given, we must therefore have some equation of the form

$$
A \lambda \mu+B \lambda+C \mu+D=0 .
$$

Any constant values for $A, B, C, D$ will be consistent with the conditions, but we can without loss of generality simplify this equation. If we make $\lambda=\mu$ we obtain the quadratic

$$
A \lambda^{2}+(B+C) \lambda+D=0 \text {. }
$$

We thus see that there are in general two critical points on the line corresponding to the roots of this equation. If we choose these two points for $x$ and $y$, which is of course possible without sacrifice of generality, the roots of this equation should be 0 and $\infty$, or in other words the constants $A$ and $D$ must be each zero. We thus see that by an appropriate choice of the fundamental points the relation between $\lambda$ and $\mu$ assumes the simple type

or, finally,

$$
B \lambda+C \mu=\text { o, }
$$

$$
\lambda=h \mu,
$$

in which $h$ is a function of the particular distance between $\lambda$ and $\mu$.

We have, however, seen that the distance is also to be expressed in the form

$$
\phi(\lambda)-\phi(\mu) .
$$

This must therefore be a function of $h$, that is, of $\lambda \div \mu$, and thus we have

$$
\phi(\lambda)-\phi(\mu)=F\left(\frac{\lambda}{\mu}\right)
$$

From this equation the particular value of the distance has disappeared. It must therefore be true for all values of $\lambda$ and all values of $\mu$. It must remain true if differentiated either with respect to $\lambda$ or $\mu$. We therefore have

$$
\begin{gathered}
\phi^{\prime}(\lambda)=\frac{\mathrm{I}}{\mu} F^{\prime}\left(\frac{\lambda}{\mu}\right) \\
-\phi^{\prime} \mu=-\frac{\lambda}{\mu^{2}} F^{\prime}\left(\frac{\lambda}{\mu}\right),
\end{gathered}
$$

whence

$$
\lambda \phi^{\prime}(\lambda)=\mu \phi^{\prime}(\mu),
$$

but as $\lambda$ and $\mu$ are independent this requires

$$
\phi^{\prime}(\lambda)=\frac{H}{\lambda}
$$

or

$$
\phi(\lambda)=H \log \lambda,
$$

whence finally we see that the distance between the two points $\lambda$ and $\mu$ is

where $H$ is a constant.

$$
H \log \frac{\lambda}{\mu},
$$

There seems nothing arbitrary in this process. We have set out with the two laws I. and II., and we have without any other assumption been conducted to the logarithmic conception of distance which lies at the foundation of the elliptic geometry. We might almost be tempted to ask how any other conception of distance can be reasonable. The two laws assumed are obviously true on any intelligible conception of distance, and yet they conduct to the logarithmic expression and apparently to nothing else.

It remains to show where the assumption made in ordinary geometry comes in. Hitherto we have not restricted the generality of the constants $A, B, C, D$ which enter into the equation between $\lambda$ and $\mu$. Euclid, however, demands that the expression

$$
(B+C)^{2}-4 A C
$$

shall be equal to zero. This has the effect of rendering the quadratic equation a perfect square. The logarithmic theory is accordingly evanescent, and we have to resort to the specialised conception of ordinary distance.

ROBEKT S. BALL

\section{NOTES}

THE Council of the Royal Society at their last meeting awarded the Copley Medal to Auguste Kekulé, of Bonn (For. Mem.R.S.), for his researches in organic chemistry, and the Davy Medal to Jean Servais Stas, of Brussels (For.Mem.R.S.), for his researches on the atomic weights. At the same meeting, Prof. D. E. Hughes, F.R.S., and Prof. E. Ray Lankester, F.R.S., were nominated for the Royal Medals-the former eminent for his electric researches, and the latter for his services to embryology and animal morphology. Her Majesty has since signified her approval of these nominations. 